\title{
CONDIÇÕES ASSOCIADAS À QUALIDADE DE VIDA DOS IDOSOS COM DOENÇA CRÔNICA*
}

Betina Hörner Schlindwein Meirelles ${ }^{1}$, Cecília Arruda ${ }^{2}$, Eunice Simon ${ }^{3}$, Fernanda Meneguello Arzuaga Vieira ${ }^{4}$, Mari Dalva Vossgrau Cortezi ${ }^{5}$, Maria Salete Lopes Natividade ${ }^{6}$

RESUMO: Estudo qualitativo-descritivo que teve por objetivo descrever as condições associadas à qualidade de vida dos idosos com doença crônica de uma comunidade pesqueira de Santa Catarina. Participaram 22 pessoas, com mais de sessenta anos, portadoras de diabetes Mellitus, hipertensão arterial, depressão e artrite/artrose. Utilizou-se para coleta de dados a entrevista semiestruturada e o instrumento WHOQOL-BREF, entre abril e agosto de 2006. A análise dos dados foi temática, utilizando o software Atlas-Ti, formando categorias segundo domínios apontados pela OMS em seus instrumentos de avaliação da qualidade de vida. Assim, o estudo resultou em duas categorias: Conceituando qualidade de vida física, social, psicológica e ambiental; e Fatores que influenciam a qualidade de vida dos idosos com doença crônica. As pessoas idosas e com doenças crônicas dão ênfase ao domínio físico, sem se esquecer da interferência dos demais domínios. Existe uma preocupação com a autonomia, que deve ser estimulada pelos profissionais de enfermagem e saúde.

PALAVRAS-CHAVE: Qualidade de vida; Doença crônica; Enfermagem; Idoso.

\section{CONDITIONS ASSOCIATED WITH THE QUALITY OF LIFE OF ELDERLY WITH CHRONIC DISEASE}

\begin{abstract}
A qualitative descriptive study that aimed to describe the conditions associated with the quality of life of elderly with chronic illness of a fishing community of Santa Catarina. Participants were 22 people with more than sixty years, suffering from diabetes mellitus, hypertension, depression and arthritis/osteoarthritis. Both WHOQOL-BREF and a semistructured interview instrument were used for data collection, between April and August 2006. A thematic data analysis was conducted with the software Atlas-Ti, forming categories on areas highlighted by the WHO in its instruments to assess quality of life. Thus, the analysis resulted in two categories: Conceptualizing physical, social, psychological and environmental quality of life, and Factors influencing quality of life of elderly with chronic disease. The elderly and people with chronic diseases emphasize the physical domain, without forgetting the interference of other areas. There is a concern for autonomy, which must be stimulated by nursing and health professionals.
\end{abstract}

KEYWORDS: Quality of life; Chronic disease; Nursing; Elderly.

\section{CONDICIONES ASOCIADAS A LA CUALIDAD DE VIDA DE LOS ANCIANOS CON ENFERMEDAD CRÓNICA}

RESUMEN: Estudio cualitativo descriptivo que tuvo por objetivo describir las condiciones asociadas a la cualidad de vida de los ancianos con enfermedad crónica de una comunidad pesquera de Santa Catarina. Han participado 22 personas, con más de 60 años, portadoras de diabetes Mellitus, hipertensión arterial, depresión y artritis/artrosis. Fueron utilizados para recoger datos la entrevista semiestructurada y el instrumento WHOQOL-BREF, entre abril y agosto de 2006. El análisis de los datos fue temático, utilizando el software Atlas-Ti, con categorias según dominios apuntados por OMS en sus instrumentos de evaluación de cualidad de vida. Así, el estudio ha resultado en dos categorías: Concepto de cualidad de vida física, social, psicológica y ambiental; y Factores que influencían la cualidad de vida de los ancianos con enfermedad crónica. Las personas mayores y con enfermedades crónicas dan énfasis al dominio físico, sin olvidarse de la interferencia de los otros dominios. Existe una preocupación con la autonomía, que debe ser estimulada por los profesionales de enfermería y salud.

PALABRAS CLAVE: Cualidad de vida; Enfermedad crónica; Enfermería; Anciano.

\footnotetext{
*Texto elaborado a partir da pesquisa "Qualidade de vida de pessoas com doença crônica", desenvolvida pelo Núcleo de Estudos e Assistência em Enfermagem e Saúde às Pessoas com Doenças Crônicas-NUCRON.

${ }^{1}$ Enfermeira. Doutora em Enfermagem. Professor Adjunto do Departamento de Enfermagem e do Programa de Pós-Graduação da Universidade Federal de Santa Cataria-PEN/UFSC. Vice-líder do NUCRON.

${ }^{2}$ Enfermeira. Mestranda em Enfermagem do PEN/UFSC. Membro do NUCRON. Bolsista CAPES.

${ }^{3}$ Enfermeira. Doutoranda em Enfermagem do PEN/UFSC. Enfermeira da Secretaria de Estado da Saúde/SC. Membro do NUCRON. ${ }^{4}$ Enfermeira. Mestre em Enfermagem. Enfermeira da Secretaria Municipal de Saúde de Balneário Camboriu/SC. Membro do NUCRON. ${ }^{5}$ Enfermeira. Mestre em Saúde Coletiva. Enfermeira da Secretaria Municipal de Saúde de Balneário Camboriu/SC. Membro do NUCRON. ${ }^{6}$ Enfermeira. Mestre em Enfermagem. Membro do NUCRON.
}

Autor correspondente:

Betina Hörner Schlindwein Meirelles

Universidade Federal de Santa Catarina

Av. Governador Irineu Bornhausen, 3188 - 88025-200 - Florianópolis-SC, Brasil

Recebido: 04/09/09

E-mail: betinam@nfr.ufsc.br

Aprovado: 26/03/10 


\section{INTRODUÇÃO}

A expressão "qualidade de vida" integra a linguagem do senso comum, como algo que as pessoas devem conquistar ou manter. A partir da década de 50, esse conceito passou a ser estudado, inicialmente pelas Ciências Sociais. No entanto, na área da saúde, o conceito vem sendo cada vez mais abordado, sendo foco de vários estudos da Organização Mundial da Saúde (OMS). Quando observamos pessoas com doenças crônicas, a qualidade de vida (QV) se coloca como algo ainda mais complexo, pois viver com uma condição crônica pode representar contínua ameaça para a pessoa, afetando sua vida como um todo. Incorporar a condição crônica representa um desafio a ser enfrentado no dia-a-dia, requerendo contínuos reajustes, avaliações e reavaliações devido à dinamicidade de sua apresentação e evolução ${ }^{(1-2)}$.

QV pode ser definida como um conceito mais genérico ou ser relacionada à saúde. Como um conceito mais genérico, foi influenciado por estudos sociológicos e é representado pelo conceito da Organização Mundial da Saúde (OMS) como "a percepção do indivíduo de sua posição na vida no contexto da cultura e sistema de valores nos quais ele vive e em relação aos seus objetivos, expectativas, padrões e preocupações"(3:1405). Consideramos QV um conceito relacionado à saúde quando se encontra associado às enfermidades ou às intervenções em saúde. Utilizou-se como referência para este estudo o conceito da OMS, no qual QV envolve algumas características como subjetividade, percepção de si, satisfação e insatisfação, mas com visão de que há condições externas que influenciam a QV das mesmas; multidimensionalidade, incluindo seu estado físico, cognitivo, afetivo e suas relações interpessoais e os papéis sociais em suas vidas e bipolaridade que inclui elementos positivos e negativos ${ }^{(3)}$.

$\mathrm{O}$ envelhecimento populacional associado à tendência ao aumento das doenças crônicas tem se tornado um grande desafio para a saúde pública em países como o Brasil, que apresentam uma grande disparidade socioeconômica. Uma maior longevidade expõe os indivíduos por mais tempo a fatores de risco como doenças crônico-degenerativas, que podem afetar a QV.

O aumento da representatividade dos idosos traz impactos diretos na alocação de recursos para o atendimento em saúde. Paulatinamente, as doenças infecto-contagiosas, mais associadas à população jovem e cujo tempo de duração é reduzido (devido à dicotomia cura ou morte), passam a dar lugar a uma crescente prevalência de condições crônico-degenerativas, caracterizadas por um prolongado tempo de doença e por requererem uma série específica de amplos cuidados em saúde( ${ }^{(4)}$.

Além de investigar e determinar o ritmo de envelhecimento populacional, existe também a preocupação de estudar a qualidade do envelhecimento e, em um segundo momento, desenhar intervenções capazes de provocar impacto em direção a um processo de envelhecimento saudável( ${ }^{(4)}$.

Neste sentido, as metas são promover a saúde e o bem-estar durante toda a vida do indivíduo. $\mathrm{O}$ envelhecimento bem sucedido é considerado como produto de uma condição de bem-estar físico e social, individual e de grupo, sendo levados em conta os valores existentes no ambiente em que o indivíduo envelhece e as circunstâncias de sua história pessoal e de seu grupo etário. Assim, observa-se uma preocupação com o processo de envelhecimento e, pesquisar QV neste contexto torna-se relevante, uma vez que valoriza questões subjetivas das pessoas, possibilitando adequar intervenções específicas de saúde para esta população.

A compreensão sobre a QV das pessoas vincula-se ao cotidiano dos serviços de saúde, influenciando decisões e condutas terapêuticas das equipes de saúde ${ }^{(5)}$. Neste sentido, salienta-se a importância da ação dos profissionais de saúde, em especial dos enfermeiros, em discutir estas questões junto à sua clientela, estimulando outros indivíduos a terem uma vida mais prazerosa, feliz e qualificada na velhice. "A qualidade do envelhecimento está diretamente relacionada com a qualidade de saúde que o indivíduo tem no seu percurso existencial e o estilo de vida que ele assume nessa trajetória"(6:253).

Considerando as dificuldades que os profissionais encontram em ampliar o conceito de saúde que ultrapasse a ausência de doenças e fortalece a QV, talvez um ponto de partida possa ser a superação do modelo tradicional de atenção às pessoas em condições crônicas de saúde; este tem sido desenvolvido através da rotina que se inicia com o diagnóstico, confirmado por exames, a definição de um tratamento básico e o acompanhamento para avaliação e ajustes que se fizerem necessários. Este modelo cristalizado vem sendo revertido a partir da valorização da subjetividade dos sujeitos e da importância da avaliação que a própria pessoa faz de sua saúde e de sua vida, e que frequentemente é melhor que a do profissional. 
Este fato é explicado pelo ajustamento à nova situação, o desenvolvimento de novas atitudes e a adequação de seu estilo de vida às limitações decorrentes da doença $^{(7)}$.

O Ministério de Saúde (MS) incorporou na política de assistência à pessoa idosa a avaliação da capacidade funcional do idoso ${ }^{(8)}$. É considerado que quanto maior o grau de dependência neste grupo etário, cuidados de maior complexidade serão requeridos, tanto da família quanto da comunidade e dos profissionais da saúde.

Diante do exposto, o objetivo deste estudo é descrever as condições associadas à QV dos idosos com doença crônica, residentes em uma comunidade pesqueira de Santa Catarina/Brasil. Trata-se de um recorte da pesquisa: "Qualidade de vida de pessoas com doença crônica de uma comunidade pesqueira do Sul do Brasil", desenvolvido pelo NUCRON, com 102 pessoas daquela comunidade, sendo que destas, 48 pessoas convivem com alguma doença crônica.

\section{METODOLOGIA}

Trata-se de um estudo descritivo, de abordagem qualitativa, desenvolvido numa comunidade pesqueira do município de Florianópolis/SC. A comunidade é bem delimitada, uma vez que seu acesso é efetuado somente por barcos ou trilha (caminhando). Apesar de distar aproximadamente quinze quilômetros do centro da cidade, o acesso da costa até a comunidade, por barco, demora cerca de 40 minutos, sendo que o transporte é regular e público. Possui uma Unidade Local de Saúde que atende a 775 habitantes.

Os integrantes da pesquisa foram 22 pessoas com mais de 60 anos de idade, selecionados entre o grupo de 48 pessoas com doenças crônicas registradas na Unidade Local de Saúde, sendo pessoas que vivem com: diabetes Mellitus, hipertensão arterial sistêmica, depressão ou doenças articulares - artrite e artrose. Os critérios de inclusão foram: ter mais de 60 anos, ser morador da comunidade há mais de um ano, ter o diagnóstico da doença crônica estabelecido há, no mínimo, um ano, comunicar-se verbalmente, estar lúcido, orientado e aceitar participar do estudo.

Os dados foram coletados no período de abril a agosto de 2006, através do instrumento abreviado de avaliação de qualidade de vida da OMS - WHOQOLBref $^{(9)}$ e entrevistas semiestruturadas, que foram previamente agendadas e realizadas no domicílio dos participantes, gravadas em áudio (com a autorização prévia dos participantes) e posteriormente transcritas. Os sujeitos foram identificados pela letra $\mathrm{P}$ seguida do número da entrevista, para garantir o anonimato.

A entrevista constou de três partes: na primeira foram levantados os dados sociodemográficos; na segunda parte foi aplicado o instrumento WHOQOLBref (versão português); e na terceira parte foram feitas perguntas semiestruturadas abordando a relação entre qualidade de vida e doença crônica. Participaram da coleta de dados pesquisadores do NUCRON, alunos de graduação e de pós-graduação do Curso de Enfermagem da UFSC.

Para a análise qualitativa de dados foi realizada a análise de conteúdo temática ${ }^{(10)} \mathrm{e}$ os dados foram organizados no software - Atlas Ti (Qualitative Research and Solutions). Este nos auxiliou na codificação dos momentos significativos evidenciados nos dados e, tendo como base o referencial teórico, buscamos a “descoberta do que está por trás dos conteúdos manifestos, indo além das aparências do que está sendo comunicado"(11:74). O software Atlas Ti permitiu-nos o primeiro nível de análise, e foi estabelecido a partir da leitura das entrevistas nas quais os dados brutos são selecionados e codificados, gerando, assim, as categorias prévias de análise. As categorias foram formadas considerando os domínios apontados pela OMS em seus instrumentos de avaliação da QV - WHOQOL$\mathrm{Bref}^{(9)}$, ou seja, domínios: físico, psicológico, das relações sociais e do meio ambiente.

A partir da análise dos dados obtiveram-se duas categorias: Conceituando qualidade de vida física, social, psicológica e ambiental, e Fatores que influenciam a qualidade de vida dos idosos com doença crônica, discutindo em especial as doenças crônicas e as possibilidades de melhoria para a qualidade de vida.

A pesquisa obedeceu a Resolução n. 196/96 ${ }^{(8)}$ e suas complementares, do CNS/MS, que dispõem sobre Diretrizes e Normas regulamentares na Pesquisa com Seres Humanos, especialmente no que diz respeito ao consentimento livre e esclarecido, sendo aprovada pelo Comitê de Ética em Pesquisa com Seres Humanos da UFSC, processo n. 069/2004.

\section{RESULTADOS E DISCUSSÃO}

A partir da análise dos dados, discute-se a seguir os resultados obtidos na pesquisa relacionados ao perfil dos participantes, aos conceitos e aos fatores que interferem na QV dos idosos com doença crônica da comunidade estudada. 
Quanto ao perfil dos 22 participantes da pesquisa, constatou-se que a idade dos sujeitos variou de 62 a 88 anos, sendo 7 homens e 15 mulheres; as doenças mais prevalentes foram hipertensão arterial, com 12 ocorrências (54,54 \%), seguida da associação diabetes e hipertensão arterial, com seis sujeitos $(27,27 \%)$.

\section{Conceituando Qualidade de vida física, social, psicológica e ambiental}

O conceito QV tem significados abrangentes. Estes podem refletir valores e uma soma de conhecimentos definidos pelos sujeitos. A partir da definição da OMS, alguns aspectos principais podem se relacionar: saúde física, psicológica, social, meio ambiente, independência e espiritualidade.

Em nosso estudo, o conceito de qualidade de vida física, atribuído por pessoas com mais de 60 anos, está relacionado ao trabalho, sendo este considerado como um aspecto importante.

A gente ter aquilo que a gente mais precisa para viver. Tem que trabalhar para manter as coisinhas que a gente precisa [...]. (P10)

[...] é ter saúde para trabalhar, mas não tenho saúde! Então, não posso trabalhar; então, não tenho qualidade de vida. (P35)

Qualidade de vida para mim, eu acho que é a gente viver bem, ter o seu conforto... É muito bom. A gente poder comer bem, se alimentar bem. Eu entendo que qualidade de vida para mim é isso. (P44)

Nestas falas, a qualidade de vida está focada na energia e no trabalho, pois depende de condições necessárias para obtê-la, como possibilidade do indivíduo ter capacidade física. A pessoa poderá satisfazê-la considerando os agravos de saúde agudos ou crônicos que refletem diretamente na $\mathrm{QV}^{(12)}$. Dessa forma, também são referidos os bens adquiridos através do trabalho - que proporcionam conforto e alimentação adequada - fatores estes que propiciam $\mathrm{QV}$ e constroem este conceito.

O conceito de QV social, identificado na fala dos sujeitos, mostra a dimensão das relações pessoais e sociais. A maneira de como compreendem e conceituam QV é expresso de forma simplificada como: Viver Bem.
Viver bem com a familia, viver bem consigo mesmo, viver com o pessoal da comunidade, ser uma boa pessoa para todo mundo gostar da gente. Qualidade de vida para mim é isso. (P34)

Eu entendo por qualidade de vida a vida que a gente leva, o dia a dia. Viver bem com o marido. Os filhos virem nos visitar. Todos os dias eles ligam para saber como a gente está; é uma preocupação a menos que a gente tem. (P23)

As relações sociais construídas pelos sujeitos, seja com a família, amigos ou vizinhos, ao longo da vida, proporcionam o bem-estar psicológico e a saúde mental. Contatos sociais, afetos positivos e intercâmbios sociais são descritos como fatores preditivos de satisfação com a vida ${ }^{(13)}$.

$\mathrm{O}$ apoio social é referenciado como fator preditor para melhor $\mathrm{QV}^{(14)}$. Simboliza o fato de a pessoa idosa possuir satisfação com a sua vida, apesar da presença da doença, porque se fundamenta na relação pessoal, familiar e social estabelecida por ela em seu contexto, construído ao longo de sua história. Fica evidenciada, assim, a importância da proximidade da família, o que contribui para o enfrentamento das adversidades cotidianas.

A família e as relações sociais de apoio apresentam-se como ponto de referência nas diversas situações e em todos os períodos da vida. Portanto, o Viver Bem conceituado pelos sujeitos nos permite olhar a velhice sob perspectiva diferente e indica a possibilidade de receber ajuda e de promover o próprio bem-estar.

O conceito de QV psicológica permeia diversas dimensões globais, sem significar ausência de problemas, presença ou não de enfermidades, dependência ou não na vida diária, integração social, suporte familiar e independência econômica. Os elementos significativos neste conceito são representados por imagem corporal e aparência, autoestima e pensamento, aprendizagem, memória e concentração. Na fala dos sujeitos, temos que:

Quem tem saúde tem disposição, e quem não tem, como eu, depende dos outros. Eu estou dependendo dos outros para me ajudar, para fazer as coisas para mim, já que eu não tenho força para fazer as coisas. Eu não tenho atividade para fazer, as pessoas é que fazem para mim. (P35)

Mas eu já sei, sou velho, sou obrigado a me conformar. (P36) 
Qualidade de vida é conforme a gente vive. Se a gente tivesse saúde, viveria melhor. Como a gente não tem saúde, vive pior. Porque com saúde é uma coisa e com doença é outra, é diferente. (P35)

Destaca-se o sentimento de insatisfação consigo próprio, relacionado à incapacidade para realizar atividades da vida diária. Isso compromete sua autoestima, autoaceitação e constitui fator de impacto importante na sua dignidade, na medida em que sua autonomia está comprometida. Comumente, a autoestima e a autonomia podem ser afetadas pela perda de papéis e status na população idosa. A associação do viver com uma doença crônica torna-se mais um elemento significativo na diminuição da qualidade de vida para estas pessoas.

Também o conceito de QV psicológica está pontuado no próprio conceito da pessoa sobre o seu corpo e a sua aparência, quando afirma 'sou velho', o que foi percebido de maneira negativa pela postura conformista. De modo geral, a sociedade possui a imagem social da velhice como época de perdas, incapacidades, com aposentadoria insuficiente, oportunidades negadas, desqualificação tecnológica e exclusão social ${ }^{(15)}$.

Há o reconhecimento das dificuldades em viver uma condição 'pior', que caracteriza a capacidade de estabelecer avaliação subjetiva sobre sua pessoa e demonstra capacidade cognitiva e potencial para tomar decisões.

Ao responderem a questão que conceitua a qualidade de vida ambiental, os sujeitos evidenciaram fortemente os fatos de ter conforto e acessibilidade ao serviço de saúde:

Qualidade de vida pra nós é conforto [...] é ter acesso integral ao Posto de saúde [à noite]. (P43)

As pessoas expressaram nas falas sua compreensão de QV e provável condição para obtê-la, frente à preocupação de viverem em uma comunidade que conta com uma Unidade Básica de Saúde, que presta atendimento somente no período diurno, associado ao fato do difícil acesso aos outros recursos de saúde, em caso de agravamento da sua condição.

Quanto às questões de acesso aos serviços de saúde, é preciso considerar a vulnerabilidade do indivíduo idoso, portador de doença crônica, e o sentimento de desamparo quanto ao tratamento/acompanhamento. Embora existam programas específicos subsidiados pelo MS, através do Sistema Único de Saúde, estes não garantem a qualidade e acesso aos serviços, o que poderá influenciar na qualidade de vida.

Os conceitos QV física, social, psicológica e ambiental, conforme definidos pelos sujeitos, sugerem a dimensão da saúde que está relacionada com a QV, o que é sugestivo de uma abordagem interdisciplinar para a promoção de saúde destas pessoas. A disponibilidade de uma equipe treinada e multiprofissional se faz necessária para o suporte terapêutico ideal desta população.

\section{Fatores que influenciam a qualidade de vida dos idosos com doença crônica}

Em relação à qualidade de vida física, as principais queixas foram associadas à condição crônica de saúde e à idade avançada. A dor física, dependência dos medicamentos, limitação nas atividades diárias, na locomoção e modificações na alimentação foram citados como influentes na QV.

A dor física é comumente associada à senilidade, que desencadeia a rigidez dos membros, restringindo a movimentação destas pessoas. $\mathrm{O}$ envelhecimento traz como consequência a diminuição do desempenho motor na realização das atividades da vida diária. No entanto, não leva as pessoas a se tornarem necessariamente dependentes de outros ${ }^{(16)}$ :

[...] a dor de cabeça é da pressão alta, [...] a dor de cabeça é da vista. (P8)

[...] a dor nas pernas, esse braço aqui está duro, o dedo fica duro. A gente tem que ficar mais sentado [...]. O cara vai ficando velho: ontem eu não tinha nada, hoje eu estou com uma dor no braço, na cabeça, vai tomando um remedinho aqui, amanhã aparece de novo! O que vai fazer? Tem que se conformar. (P39)

Algumas condições crônicas, como a hipertensão arterial, podem gerar episódios de diminuição da acuidade visual causando dores de cabeça relevantes e assim, influenciando no bem-estar.

Os participantes mostraram-se conscientes da importância e necessidade de seguir o tratamento medicamentoso de forma correta, principalmente aqueles que sofreram experiências negativas ao não estimar a prescrição médica. Muitos se veem dependentes do uso da medicação para o controle da doença crônica e alívio das dores recorrentes: 
Olha, se eu não tomar hoje o remédio, eu me acho mal. Então, todo dia de manhã eu tomo o remédio. Tomo o do coração, o da pressão e o da labirintite. Todo dia eu tenho que tomar esses três remedinhos [...]. No ano passado chegava a cair por causa da pressão. (P27)

[...] só tomar o remédio para a gente ter bastante saúde, é o jeito. (P11)

Buscar o tratamento, avaliar o resultado do uso de medicamentos, realizar a terapia, seguir a dieta e a realização de atividade física são maneiras que possibilitam QV às pessoas com doenças crônicas ${ }^{(15)}$.

A dependência parcial ou total de outras pessoas (especialmente familiares) e a utilização de equipamentos auxiliares para locomoção muitas vezes torna-se necessária, evidenciando a situação debilitada da pessoa senil e em condição crônica de saúde:

Isso ai me atrapalha [usar bengala], porque a gente tem vontade de se movimentar, de se mover, e não pode. Tudo atrapalha. (P35)

A maior influência do domínio físico na qualidade de vida global desses idosos ressalta a importância de se considerar a capacidade funcional como importante fator de impacto na QV em idosos ${ }^{(17)}$.

Quanto à QV social, dois aspectos foram destacados pelos participantes. O primeiro faz menção às relações pessoais, ressaltando que limitações na vida diária - não poder passear ou não participar das atividades da comunidade devido à diminuição da acuidade visual e dificuldade de movimentação promovem dependência e/ou isolamento, provocando limitação social. Como segundo aspecto, destaca-se a desestruturação do suporte social e familiar. Alguns participantes relataram que a dificuldade no relacionamento familiar colabora para o desenvolvimento de doenças ou agravo de sua condição crônica:

Estou me sentindo bem. É só ter confusão e briga dentro de casa. Eu acho que é o que causou esse problema comigo. É uma coisa assim: tu ficas mais deprimida, tu ficas com a pressão alta... Porque eu não gosto desse tipo de coisa. Eu não posso ver isso. (P44)

No estudo acerca dos fatores associados ao envelhecimento bem-sucedido de idosos socialmente ativos da região metropolitana de Porto Alegre, percebe-se que:

\begin{abstract}
A manutenção da independência para as atividades da vida diária, autonomia e satisfação com relacionamento familiar e amizades foram fatores preditivos independentes do envelhecimento bem-sucedido, tanto para homens como para mulheres. Conforto material, sentirse fisicamente bem, imagem corporal e aparência, autoestima, sentimentos positivos, relações interpessoais, suporte social, participação em atividades recreativas, sexualidade, espiritualidade e crenças foram preditivos para as mulheres. O idoso é um ser de relação consigo mesmo, com os outros e com as suas crenças. A família é o principal suporte social ${ }^{(18: 302-308)}$.
\end{abstract}

Com relação à QV psicológica, esta apresentou significante associação com sentimentos negativos vivenciados pelos idosos em condições crônicas. Foram citados: o "medo" por ser portador de doença crônica, de não compreender a sua evolução, a incerteza quanto ao apoio futuro e a solidão. Na interpretação de suas falas, destaca-se:

Eu espero que Deus me ajude para eu viver sempre bem com os meus filhos, para os meus filhos me adotarem, porque eu estou na minha velhice, para tomar conta de mim. Para a gente não andar jogada na casa de um, na casa de outro. (P27)

E ainda, em relação à qualidade de vida ambiental, as condições de moradia influenciaram diretamente neste aspecto. Ter casa própria, segurança e infraestrutura adequada acarretam pontos positivos para a manutenção da prevenção de agravos à doença e promoção da saúde. Contudo, é necessário dispor de recursos financeiros para manter e adequar o ambiente às suas necessidades:

Pois é, estar bem na minha casa. Graças a Deus não sou mandada assim, de ninguém! Hoje, eu estou na minha propriedade. (P37)

Nem sempre a gente tem as coisas aqui de casa de acordo como a gente queria que fosse [...] Ai, a gente tem que se conformar com aquilo que a gente tem em casa. Então, às vezes, a gente tem dificuldade em ter um pouco de qualidade de vida. (P44)

O negócio, hoje em dia, quem faz éo dinheiro. Se não tem o dinheiro, minha filha, passa mal! (P1)

O recurso financeiro consegue favorecer sua 
sobrevivência, contudo não possibilita uma melhor QV, pois o seu baixo poder de compra produz insatisfação.

O envelhecimento saudável passa a ser visto como uma interação multidimensional entre saúde física e mental, independência na vida diária, integração social, suporte familiar e independência econômica ${ }^{(17)}$, pois são elementos que propiciam melhor QV.

A melhoria da QV da pessoa idosa, abordada neste estudo, abrangeu uma diversidade de condições, física, social, psicológica e ambiental. Alguns recortes do discurso dessas pessoas simbolizam sua compreensão a respeito do conceito, fatores que interferem e aspectos para ter mais QV.

\section{REFLEXÕES FINAIS}

Salientamos que o conceito de QV apresentado pelos sujeitos pesquisados faz referência aos domínios preconizados pela OMS. O fato dos entrevistados serem pessoas idosas e com doenças crônicas dá ênfase ao domínio físico no discurso, sem esquecer-se da interferência dos demais domínios na QV.

Os profissionais de saúde têm o desafio de lidar com as diferentes dimensões da doença crônica, não valorizando apenas a questão biológica em si, mas trabalhando os conflitos vivenciados pelas pessoas idosas, bem como suas crenças e valores, de forma a favorecer o bem-estar e, com isso, a obtenção de melhor QV.

A preocupação com o cuidado do idoso e sua QV não se restringe apenas à família, mas suscita a discussão sobre a formação de uma rede de apoio para assistência ao idoso em um contexto multidimensional, não focando apenas nos aspectos físicos e sim se levando em conta questões psicológicas, relações sociais e meio ambiente.

Torna-se fundamental avaliar a QV sob uma variedade de dimensões, para possibilitar a abrangência dos diferentes fatores que a influenciam. Sugere-se que outros estudos sejam realizados neste sentido, a fim de complementar os resultados obtidos nesta pesquisa, considerando a importância da valorização da subjetividade de quem vive a situação e da avaliação que a própria pessoa faz de sua saúde e de sua vida.

Conhecer a percepção subjetiva das pessoas idosas acerca do conceito de $\mathrm{QV}$, e os fatores que interferem para conquistá-la ou mantê-la, é necessário aos profissionais de saúde e/ou cuidadores para o planejamento do cuidado individual/coletivo, com o intuito de minimizar o sofrimento humano e garantir/ proporcionar melhor qualidade de vida.

\section{REFERÊNCIAS}

1. Hymovich DP, Hagopian G. Chronic illness in children and adults: a psychosocial approach. Philadelphia: Saunders; 1992.

2. Silva DGV, Koschnik Z, Azevedo MA, Souza SS. Qualidade de vida de pessoas com insuficiência renal crônica em tratamento hemodialítico. Rev Bras Enferm. 2002;55(5):562-7.

3. The WHOQOL Group. The World Health Organization quality of life assessment (WHOQOL): position paper from the World Health Organization. Social Science \& Medicine. 1995;41(10):1403-9.

4. Chachamovich E. Qualidade de vida em idosos e o processo de envelhecimento [projeto de tese]. Porto Alegre: Universidade Federal do Rio Grande do Sul; 2006.

5. Seidl EMF, Zannon CMLC. Qualidade de vida e saúde: aspectos conceituais e metodológicos. Cad Saúde Pública. 2004;20(2):580-7.

6. Celichi KLS, Spadarin G. Estilo de vida e saúde: condicionantes de um envelhecimento saudável. Cogitare Enferm. 2008;3(2) 252-60.

7. Ramos N, Crepalde ATA. Qualidade de vida em doenças pulmonares crônicas: aspectos conceituais e metodológicos. J Pneumol. 2000;26(4):207-13.

8. Ministério da Saúde (BR). Envelhecimento e saúde da pessoa idosa. Caderno de Atenção Básica n. 19. Brasíla, 2006.

9. Fleck MPA, Louzada S, Xavier M, Chachamovich E, Vieira G, Santos L, et al. Aplicação da versão em português do instrumento abreviado de avaliação da qualidade de vida "WHOQOL-bref". Rev Saúde Pública. [Internet] 2000;34(2) [acesso em 22 nov 2007]. Disponível: http://tiny.cc/5eb0b

10. Minayo MCS. Pesquisa social: teoria, método e criatividade. Petrópolis: Vozes; 1997.

11. Minayo MCS. O desafio do conhecimento: metodologia da pesquisa social em saúde. Rio de Janeiro: HUCITEC/ABRASCO; 1994.

12. Scattolin FA, Diogo MJDE, Colombo RCR. Correlação entre instrumentos de qualidade de vida relacionada à saúde e independência funcional em idosos com insuficiência cardíaca. Cad Saúde Pública. [Internet] 
2007;23(11) [acesso em 22 nov 2007]. Disponível: http://tiny.cc/jiq7j

13. Guedea MTD, Albuquerque FJB, Tróccoli BT, Noriega JAV, Seabra MAB, Guedea RLD. Relação do bem-estar subjetivo, estratégias de enfrentamento e apoio social em idosos. Psicol Reflex Crit. [Internet] 2006;19(22) [acesso em 22 nov 2007]. Disponível: http://tiny.cc/ax834

14. Rudnicki T. Preditores de qualidade de vida em pacientes renais crônicos. Estud Psicol. [Internet] 2007;24(3) [acesso em 22 nov 2007]. Disponível: http:// tiny.cc/u8amp

15. Paschoal SMP. Qualidade de vida do idoso: elaboração de um instrumento que privilegia sua opinião [dissertação]. São Paulo(SP): Universidade de São Paulo; 2000.

16. Andreotti RA, Okuma SS. Validação de uma bateria de testes de atividades da vida diária para idosos fisicamente independentes. Rev Paul Educ Fís.1999;13(1):46-66.

17. Pereira RJ, Cotta RMM, Franceschini SCC, Ribeiro RCL, Sampaio RF, Priore SE, et al. Contribuição dos domínios físico, social, psicológico e ambiental para a qualidade de vida global de idosos. Rev Psiquiatr. [Internet] 2006;28(1). [acesso em 26 nov 2007]. Disponível: http://tiny.cc/qy1n5

18. Moraes JFD, Souza VBA. Fatores associados ao envelhecimento bem-sucedido de idosos socialmente ativos da região metropolitana de Porto Alegre. Rev Bras Psiquiatr. [Internet] 2005;27(4) [acesso em 25 out 2007]. Disponível: http://tiny.cc/oc3ry 